Note et information.

\title{
HYALOMMA SCUPENSE SCHULZE, 1919 (ACARINA, IXODOIDEA) TIQUE AUTOCHTONE DU SUD-OUEST DE LA FRANCE
}

\author{
F. MACAIGNE *, C. PEREZ-EID **
}

RÉSUMÉ

Les auteurs signalent la présence en Aquitaine de Hyalomma scupense en tant qu'espèce autochtone.

Summary: Hyalomma scupense Schulze, 1919 (Acarina, Ixodoidea) autochthon tick from South-West France.

The authors report the presence of autochthon Hyalomma scupense collected from cattle of the South-West of France.

\section{INTRODUCTION}

L'inventaire des tiques de France métropolitaine peut être actuellement considéré comme quasi définitif. Sénevet (13) signalait 16 espèces en 1935. Plus récemment, Rageau (12), indiquait la présence de 36 taxa. Depuis lors, d'autres espèces sont venues s'ajouter à cette liste en particulier Pholeoixodes rugicollis (2), Ixodes ventalloi longtemps confondu avec I. festai (6), Argas macrostigmatus (7) et I. apronophorus (5). Jusqu'à maintenant, peu de travaux ont été consacrés aux tiques du genre Hyalomma récoltées en France. Lamontellerie (8), en 1954, signalait la présence en Charente-Maritime [17], en Gironde [33] et dans le département des Landes [40] de Hyalomma savignyi (Gervais, 1844) synonyme de $H$. marginatum marginatum Koch, 1844. En 1959 Morel (10), sur la vue d'une photographie de la tique (8), pensait qu'il s'agissait plutôt de $H$. excavatum Koch, 1844 synonyme de $H$. anatolicum Koch, 1844 et de $H$. anatolicum excavatum Koch, 1844 ; Pomerantzev, 1946. Un nouvel examen des tiques faisait alors conclure à Lamontellerie (9) que ses identifications antérieures étaient incorrectes et que l'espèce étudiée était en fait $H$. scupense Schulze, 1919. Récemment la présence de ce taxon dans le sud-ouest de la France a été encore une fois remise en question par Couatarmanac'h et coll. (4).

Nous nous sommes donc proposés d'examiner à nouveau les tiques provenant de collectes réalisées par Lamontellerie et d'y ajouter quelques exemplaires provenant de

* Laboratoire de Parasitologie et de Mycologie Médicales, Université de Bordeaux II, 146, rue Léo-Saignat, F 33076 Bordeaux Cedex.

** Unité d'Écologie des Systèmes Vectoriels, Institut Pasteur, 28, rue du Docteur Roux, F 75724 Paris Cedex 15.

Accepté le : 23 mars 1993. récoltes personnelles afin de conclure définitivement sur l'identité de ces Hyalomma.

\section{MATÉRIEL ET MÉTHODES}

60 spécimens, tous prélevés sur bovins, ont été examinés :

— Récolte de février 1952 : 18 femelles et 4 mâles à Morcenx (Landes).

- Récolte d'avril 1952 : 3 femelles et 1 mâle à Morcenx (Landes).

- Récolte d'avril 1953 : 10 femelles à Arengosse (Landes).

- Récolte d'avril 1953: 18 femelles et 2 mâles à Gujan-Mestras (Gironde).

- Récolte de mars 1992 : 3 femelles et 1 mâle au Verdon (Gironde).

Les gonopores des femelles ont été découpés puis montés entre lame et lamelle afin d'étudier leurs structures au microscope (1).

\section{RÉSULTATS}

Les tiques examinées appartiennent toutes à l'espèce $\mathrm{Hya}$ lomma scupense.

\section{DISCUSSION}

En Aquitaine, les tiques ont fait l'objet de peu de collectes. Coléno (3), en 1924, signalait la présence de 6 espèces en Gironde : Ixodes ricinus, Pholeoixodes hexagonus, Haemaphysalis punctata, Dermacentor reticulatus, Rhipicephalus sanguineus et Argas reflexus. Un travail de plusieurs années (9) permettait à Lamontellerie d'ajouter à cette liste Eschatocephalus vespertilionis, Ixodes frontalis, I. ventalloi, Dermacentor marginatus, Haemaphysalis concinna, Rhipicephalus bursa, Carios vespertilionis, Alectorobius coniceps et Hyalomma scupense. Cette dernière espèce pré- 
sente un cycle monoxène et parasite principalement les bovins, les chevaux et les moutons. Sa morphologie est très proche de celle de $H$. detritum, ce qui a conduit Morel à ne considérer qu'une seule espèce $H$. detritum avec 2 sousespèces différentes $H$. detritum detritum et $H$. detritum scupense. Ces 2 sous-espèces différent par leurs distributions géographiques et leurs biologies. $H$. detritum detritum posséde un cycle dixène et une répartition plus méridionale que celle de $H$. detritum scupense.

L'hypothèse de Lamontellerie, selon laquelle cette dernière espèce aurait été introduite avec des chevaux par les troupes allemandes entre 1940 et 1945 , ne nous semble pas fondée. En effet, Morel signale l'existence dans la collection Brumpt d'une femelle récoltée sur un bœuf en 1933 à Samadet dans le département des Landes (11) permettant ainsi d'établir la présence de cette espèce en Aquitaine avant la deuxième guerre mondiale.

En France, $H$. scupense a une distribution pratiquement limitée, à notre connaissance, au sud-ouest. L'espèce est actuellement connue des départements suivants : CharenteMaritime [17], Gironde [33], Landes [40], PyrénéesAtlantiques [64]. Elle a également été trouvée une fois sur un cheval (11) dans la Sarthe [72].

\section{CONCLUSION}

$H$. scupense est une tique présente en France et qui doit être considérée comme bien établie en Aquitaine.

Remerciements. - Nous remercions le Docteur M. LAMONTELLERIE qui a bien voulu nous confier sa collection personnelle de Hyalomma ainsi que le Docteur P. C. MoREL pour la relecture du manuscrit et les discussions fructueuses échangées avec lui.

\section{RÉFÉRENCES}

1. Adler S., Feldman-Muhsam B. : The differenciation of ticks of the genus Hyalomma Koch in Palestine. Refuah Vet., 1946, 3, 91-94.

2. Aubert M. F. A. : Identification en France de Pholeoixodes rugicollis. (Schulze et Schlottke, 1929) et première observation des stades préimaginaux de cette espèce. Ann. Parasitol. Hum. Comp., 1974, 49, 247-248.

3. Coléno R. G. : Contribution à l'étude des Ixodidés et de leur rôle pathogène. Ixodidés de la Gironde. Thèse Médecine, Bordeaux, 1924.

4. Couatarmanac'h A., Chastel C., Chastel O., Beaucournu J. C. : Tiques d'importation observées en Bretagne. Bull. Soc. Fr. Parasitol., 1989, 7, 127-132.

5. Gilot B., Moncada E., Pautou G. : Présence en France d'Ixodes apronophorus (Schulze, 1924) (Ixodoidea, Ixodidae). Ann. Parasitol. Hum. Comp., 1976, 51, 601-603.

6. Gilot B., Perez C. : Individualisation et caractérisation de deux Ixodes actuellement confondus : I. Festai Rondelli, 1926, I. ventalloi Gil Collado, 1936 (Acarina, Ixodoidea). Rev. Suisse Zool., 1978, 85, 143-149.

7. Guiguen C. : Notes sur Argas (Argas) macrostigmatus Filippova, 1961. Bull. Soc. Fr. Parasitol., 1990, 8, 157-164.

8. Lamontellerie M. : Les Ixodoïdés du sud-ouest de la France. Espèces rencontrées, agressivité, rôle pathogène. Thèse médecine, Bordeaux, 1954.

9. Lamontellerie M. : Les tiques (Acarina, Ixodoidea) du sudouest de la France. Ann. Parasitol. Hum. Comp., 1965, 40, 87-100.

10. Morel P. C. : Les Hyalomma (acariens, Ixodidae) de France. Ann. Parasitol. Hum. Comp., 1959, 34, 552-555.

11. Morel P. C. : Les tiques d'Afrique et du bassin méditerranéen (Ixodoidea). Distribution, biologie, écologie. Document ronéotypé, Maisons-Alfort, 1964-1965.

12. Rageau J. : Répartition géographique et rôle pathogène des tiques (acariens : Argasidae et Ixodidae) en France. Wiad. Parazytol., 1972, 18, 707-719.

13. Senevet G. : Ixodoidés. Faune de France, P. Lechevallier, 1937.

Des microfiches et des microfilms de cette publication peuvent être obtenus auprès de :

This publication is available in microform from:

MASSON-SPPIF (réf. MIMC), 120, boulevard Saint-Germain, F-75280 Paris Cedex 06.

Tous droits de traduction, d'adaptation et de reproduction par tous procédés réservés pour tous pays.

Toute reproduction ou représentation intégrale ou partielle, par quelque procédé que ce soit, des pages publiées dans le présent ouvrage, faite sans l'autorisation de l'éditeur est illicite et constitue une contrefaçon. Seules sont autorisées, d'une part, les reproductions strictement réservées à l'usage du copiste et non destinées à une utilisation collective, et d'autre part, les courtes citations justifiées par le caractère scientifique ou d'information de l'œuvre dans laquelle elles sont incorporées (art. L. 122-4, L. 122-5 et L. 335-2 du Code de la propriété intellectuelle).

Des photocopies payantes peuvent être réalisées avec l'accord de l'éditeur. S'adresser au : Centre français d'exploitation du droit de copie, 3, rue Hautefeuille, 75006 Paris. Tél. 43-26-95-35.

Masson, éditeur, Paris. Imprimé par Imprimerie Barnéoud, à Laval. - 10071.

Printed in France.
Dépôt légal : 1993.

-
$\mathrm{N}^{\circ}$ d'ordre : 6413.

Commission paritaire : $\mathrm{n}^{\circ} 54169$ 